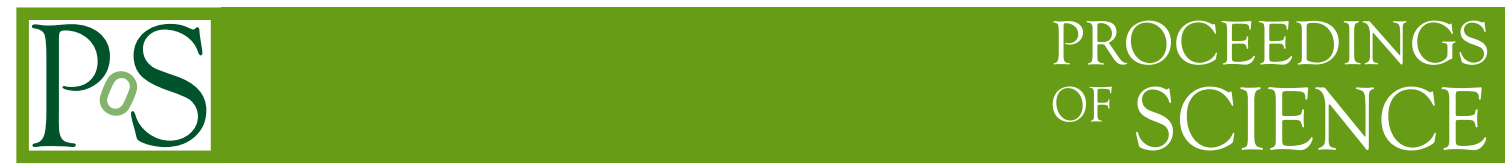

\title{
Heavy quark photoproduction at HERA
}

\section{Michel SAUTER ${ }^{* \dagger}$}

University Heidelberg, Physikalisches Institut

E-mail: michel.sauteredesy.de

The production of charm and beauty quarks in $e p$-scattering at HERA has been studied by the $\mathrm{H} 1$ and ZEUS collaborations over a wide phase space range. Data from charm and beauty photoproduction $\left(Q^{2} \approx 0 \mathrm{GeV}^{2}\right)$ are a good test for perturbative Quantum Chromodynamics. The measured heavy flavour cross sections are reasonably well described by next-to-leading order calculations.

36th International Conference on High Energy Physics,

July 4-11, 2012

Melbourne, Australia

\footnotetext{
* Speaker.

†n behalf of the H1 and ZEUS collaborations.
} 


\section{Introduction}

The various measurements of charm and beauty production at the electron-proton collider HERA, based on different experimental techniques and covering a wide phase space range, are important to test the accuracy of perturbative Quantum Chromodynamics (pQCD). In leading order (LO) QCD, the dominant contribution to heavy flavour production in $e p$-collisions, $e p \rightarrow e c \bar{c} X$, $e b \bar{b} X$, is the photon-gluon fusion (PGF) process, in which a photon emitted from the electron fuses with a gluon from the proton to a charm- or beauty-quark pair as depicted in figure 1 1 a. Resolved processes where the photon fluctuates into a hadronic state before undergoing a hard collision, as indicated in figure $11 \mathrm{~b}$ contribute to a lower extent. Predictions derived in the framework of pQCD are expected to be reliable whenever an experimental hard scale, $\mu$, is available, with $\mu$ being much larger than $\Lambda_{Q C D}$. Since the charm- and beauty-quark masses are larger than $\Lambda_{Q C D}$, this condition is fulfilled for charm or beauty production. Depending on the measurement, two additional experimental hard scales may be available: the square root of the photon virtuality, $Q^{2}$, and the transverse energy or momentum of the heavy-quark jet.

Four analyses recently released by the $\mathrm{H} 1$ and ZEUS collaborations are discussed, measuring heavy flavour production in photoproduction, where the exchanged photon is almost real $\left(Q^{2} \approx 0 \mathrm{GeV}^{2}\right)$ and does not provide a hard scale. Results on heavy flavour production in Deep-Inelastic-Scattering with $Q^{2}>0 \mathrm{GeV}^{2}$ are presented in [1] .

a)

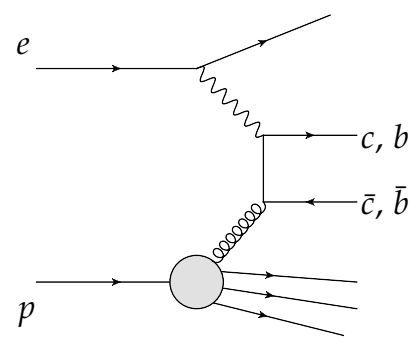

b)

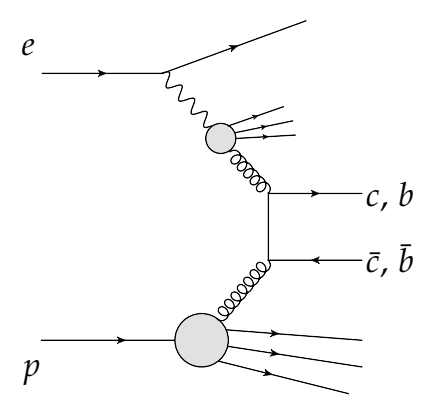

Figure 1: Generic leading order diagrams for $c \bar{c}$ or $b \bar{b}$ production in $e p$ collisions. The diagram a) is referred to as direct or pointlike, the diagram $b$ ) is referred to as resolved or hadronlike.

\section{Theoretical aspects}

Perturbative calculations of heavy-quark production at HERA are available up to next-toleading order (NLO) in $\alpha_{s}$. A number of theoretical approaches exist to describe heavy flavour quark production in $e p$-collisions. The different theoretical approximations of heavy flavour quark production are formulated in different theoretical schemes, treating the heavy quark either as massive and produced dynamically (massive scheme), or as massless and as an active constituent of the proton at large scales (massless scheme). The massive scheme is expected to describe the data better at experimental scales of the same order as the heavy quark mass. For predictions in the massive scheme the programs FMNR [2] and MC@NLO [3] are used. The massless scheme is expected 
to be valid in regions of the phase space involving larger experimental scales. The general mass variable flavour number scheme (GM VFNS) [4] for heavy quarks interpolates from the massive approach at low scales to the massless approach at high scales.

\section{Experimental aspects}

\subsection{ZEUS and H1 Detectors at HERA}

The ring collider HERA accelerated electrons and protons to energies of $E_{e} \simeq 27.6 \mathrm{GeV}$ and $E_{p} \simeq 920 \mathrm{GeV}$. Inside the two experiments $\mathrm{H} 1$ and ZEUS the two beams were brought to collision, resulting at a centre of mass energy of $\sqrt{s} \simeq 320 \mathrm{GeV}$. HERA was operated from 1992-2007 in two operation periods and delivered to each experiment a data set corresponding to an integrated luminosity of $L \simeq 0.5 \mathrm{fb}^{-1}$. H1 and ZEUS were classical, asymmetric multi-purpose detectors designed for the measurement of electron-proton interactions. A detailed description of $\mathrm{H} 1 \mathrm{can}$ be found in [5] and of ZEUS in [6].

\subsection{Experimental heavy flavour tagging methods at HERA}

In photoproduction charm and beauty production at HERA is suppressed with respect to the production of light flavoured quarks $(u, d, s)$. The corresponding cross sections and consequently event rates very roughly behave like: $\sigma_{u d s}: \sigma_{c}: \sigma_{b} \approx 2000: 50: 1$. For charm a full reconstruction of the $D$-mesons is possible. This is preferably done with so-called $D^{*}$-tags, where the $D^{*}$-meson decays to $D^{0} \pi$ and the reconstruction of subsequent $D^{0}$ decays to $K \pi$ are exploited. For beauty, however, no suitable decay channel exists which provide enough statistics when being fully reconstructed. Therefore other tagging strategies are applied, which are based on the large quark masses, the long lifetime and the large lepton-decay branching ratio, $b \rightarrow \ell+X$, of $B$-hadrons.

\section{Recent measurements}

The H1 collaboration recently released a measurement of charm photoproduction [7] based on the reconstruction of $D^{*}$-mesons already during the data record [8]. The offline analysis yields to differential cross sections of high precision, which are presented as function of several kinematic variables. The obtained data is compared to NLO pQCD predictions calculated in the massive scheme and the GM VFNS. Generally the predictions show, within their rather large uncertainties, a reasonable agreement with data. In figure 1 a the single differential cross section for $D^{*}$ production is shown as a function of the pseudo rapidity of the $D^{*}$ meson. The analysis is extended by requiring two jets in addition to the tagged $D^{*}$-meson. These additional jets introduce an additional hard scale applicable for pQCD convergence. Of particular interest of this extension is $x_{\gamma}$, which in leading order corresponds to the fraction of the photon's momentum that enters the interaction, and the azimuthal difference between the two jets $\Delta \varphi$. Using $x_{\gamma}$, interactions with a pointlike photon (direct processes) can be discriminated against processes where the photon has a resolved hadronic structure. In LO QCD a back-to-back topology is expected $(\Delta \varphi=\pi)$, while radiation of additional gluons leads to significantly smaller opening angles. Differential cross sections as function of $\Delta \varphi$ are therefore a good test for NLO calculations. In figure $2 \mathrm{~b}$ the cross section differential in $\Delta \phi$ is 
compared to the predictions of MC@NLO for the resolved enhanced phase space. In this region the NLO calculation is significantly below the data.

The H1 and ZEUS collaborations have both published simultaneous measurements of charm and beauty jets in photoproduction [9, 10]. In figure 3 the charm and beauty jet cross sections as a function of the transverse momentum of the jet are shown for the ZEUS measurement. The H1 analysis requires in addition to the two jets a muon and the heavy flavour contribution to the data set is determined from fits to the distribution of the impact parameter $\delta$ of the muon track and the relative transverse momentum $p_{\mathrm{T}}^{\text {rel }}$ of the muon with respect to the nearest jet axis. The ZEUS measurement benefits in terms of statistics from an inclusive selection and extracts the heavy flavour cross sections from distributions of displaced tracks and from secondary vertex masses. The two measurements agree with the NLO pQCD predictions as calculated with the program FMNR or MC@NLO.

In another measurement the [11] $\mathrm{H} 1$ collaboration has measured beauty photoproduction near threshold, by reconstructing beauty quarks at lowest transverse momenta, i.e. in a phase space region where the only available hard scale is the mass of the beauty quark. This is achieved by using the decay channel $b \bar{b} \rightarrow e e X$. Low $p_{\mathrm{T}}$ electrons were identified on trigger level [8, 12], and offline via a dedicated electron reconstruction algorithm having an excellent efficiency and background rejection behaviour. The beauty signal is discriminated against background by exploiting di-electron correlations based on the invariant di-electron mass, the azimuthal angle between the electrons, and product of the electron charges. The unfolded differential cross section as function of the mean transverse momentum of the beauty-quark pair is compared to the massive NLO-QCD prediction from FMNR.

In Figure 4 the differential cross sections of beauty photoproduction of the discussed measurements are compared to all currently available beauty photoproduction measurements at HERA and the massive NLO pQCD prediction. It can be seen that the prediction agrees in general rather well with the data, which confirm each other over a wide range in $p_{\mathrm{T}}^{\mathrm{b}}$. Note that for the central value of the pQCD prediction for the renormalization and factorization scales to be $\mu_{r, f}=\mu_{0}=1 / 2 \sqrt{m_{b}^{2}+p_{\mathrm{T}}^{2}}$, while previous predictions usually were based on twice this value [13].

\section{Conclusion}

Heavy flavour photoproduction at HERA is intensively studied in many analyses with various heavy flavour tagging methods, achieving high experimental precision and agreeing each other over a large range in phase space. The heavy flavour production measurements are able to test $\mathrm{QQCD}$ at different scales. In general the discussed $\mathrm{pQCD}$ calculations describe the data reasonably well in shape and normalization.

\section{References}

[1] A. Geiser these proceedings.

[2] S. Frixione, M. L. Mangano, P. Nason and G. Ridolfi, Adv. Ser. Direct. High Energy Phys. 15 (1998) 609 [hep-ph/9702287].

S. Frixione, P. Nason and G. Ridolfi, Nucl. Phys. B 454 (1995) 3, [hep-ph/9506226]. 
S. Frixione, M. L. Mangano, P. Nason and G. Ridolfi, Phys. Lett. B 348 (1995) 633, [hep-ph/9412348].

[3] S. Frixione and B.R. Webber, JHEP 0206 (2002) 029 [hep-ph/0204244];

S. Frixione, P. Nason and B.R. Webber, JHEP 0308 (2003) 007 [hep-ph/0305252].

T. Toll and S. Frixione, Phys. Lett. B703 (2011) 452 [arXiv:1106.1614].

[4] G. Kramer and H. Spiesberger, Eur. Phys. J. 38 (2004) 309 [hep-ph/0311062].

B.A. Kniehl, G. Kramer, I. Schienbein and H. Spiesberger, Eur. Phys. J. 62 (2009) 365 [arXiv:0902.3166].

[5] I. Abt et al. [H1 Collaboration], Nucl. Instrum. Meth. A 386 (1997) 310;

I. Abt et al. [H1 Collaboration], Nucl. Instrum. Meth. A 386 (1997) 348.

[6] ZEUS Coll., U. Holm et al., The ZEUS Detector. Status Report (unpublished), DESY (1993), available on http://www-zeus.desy.de/bluebook/bluebook.html

[7] F. D. Aaron et al. [H1 Collaboration], Eur. Phys. J. C 72 (2012) 1995 [arXiv:1203.1170].

[8] A. W. Jung et al., Proc. 15th IEEE-NPSS Real-Time Conference, (2007) 1.

[9] F. D. Aaron et al. [H1 Collaboration], Eur. Phys. J. C 72 (2012) 2047 [arXiv:1205.2495].

[10] H. Abramowicz et al. [ZEUS Collaboration], Eur. Phys. J. C 71 (2011) 1659 [arXiv:1104.5444].

[11] F. D. Aaron et al. [H1 Collaboration], Eur. Phys. J. C 72 (2012) 2148 [arXiv:1206.4346].

[12] B. Olivier et al. Nucl. Instrum. Meth. A 641 (2011) 58.

[13] A. Geiser, Nucl. Phys. Proc. Suppl. 184 (2008) 189.

a)

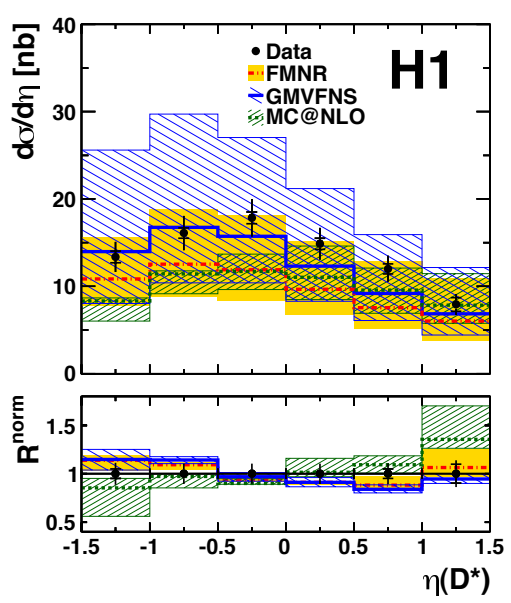

b)

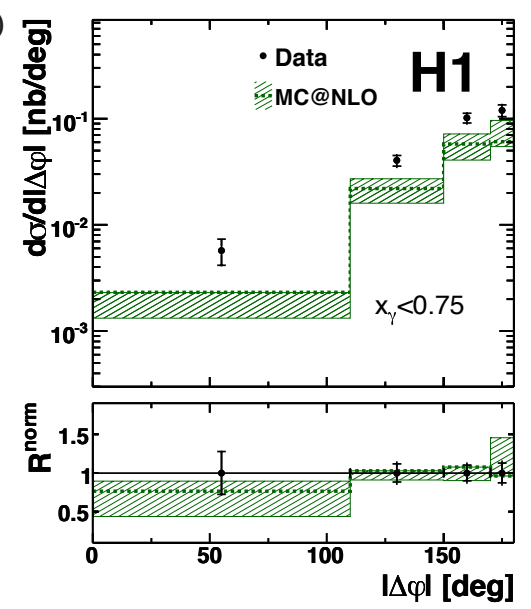

Figure 2: Left: Single differential $D^{*}$ cross section as a function of $\eta\left(D^{*}\right)$ compared to the NLO predictions of FMNR, GM VFNS and MC@NLO. Right: Single differential cross section for $D^{*}$-tagged dijet production as a function of the difference in in azimuthal angle $\Delta \varphi$ between the other jet and the $D^{*}$ jet compared to MC@NLO predictions. The data are shown as points with the inner errors being the statistical errors and the outer errors the total uncertainty. The bands indicate the NLO predictions including the theoretical uncertainty. The normalised ratio $\mathrm{R}^{\text {norm }}$ is shown as well. 


\section{ZEUS}
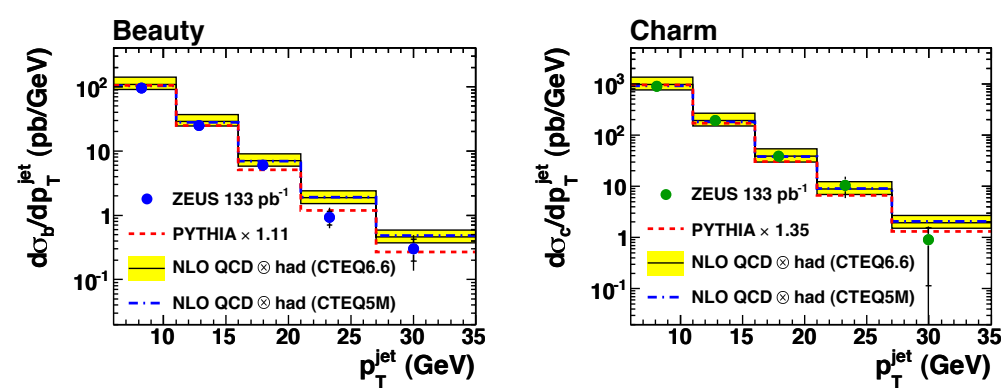

Figure 3: Differential beauty-jet and charm-jet photoproduction cross sections of [10] as a function of $p_{\mathrm{T}}^{\text {jet }}$. The data are shown as points. The inner error bars are the statistical uncertainties, while the outer error bars show the statistical and systematic uncertainties added in quadrature. The band represents the NLO QCD prediction, the shaded band shows the estimated uncertainty. The scaled LO PYTHIA MC prediction (dashed line) is also shown.

\section{HERA}

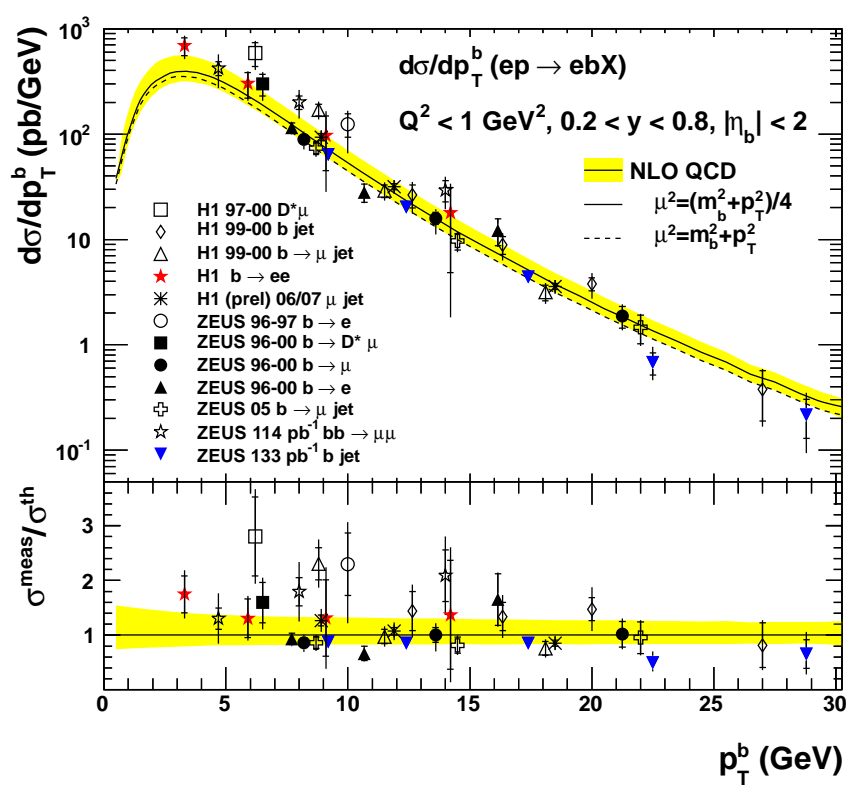

Figure 4: Upper plot: Compilation of beauty photoproduction cross section measurements as function of the mean transverse momentum of the beauty-quark (points), compared to a massive NLO pQCD calculation [2] (shaded band). The discussed measurements [10, 11] are shown in blue and red. Lower plot: Ratio of the measured cross sections and the calculated NLO pQCD prediction. 